\title{
Influência de agrupamentos de bambu na dinâmica pós-fogo da vegetação lenhosa de um cerrado típico, Mato Grosso, Brasil
}

Influence of bamboo clumps in post-fire dynamics of the woody vegetation of a typical cerrado, Mato Grosso, Brazil

\author{
Henrique Augusto Mews ${ }^{1}$, Divino Vicente Silvério ${ }^{2}$, Eddie Lenza ${ }^{3}$ \& Beatriz Schwantes Marimon ${ }^{3,4}$
}

\begin{abstract}
Resumo
Neste estudo foram avaliadas as mudanças na composição florística e na estrutura da vegetação lenhosa logo após incêndio acidental ocorrido em sítios de cerrado típico sem (SB, controle) e com (CB) agrupamentos do bambu Actinocladum verticillatum em Nova Xavantina/MT. Foram amostrados todos os indivíduos com diâmetro de base $\left(\mathrm{Db}_{30}\right) \geq 3 \mathrm{~cm}$ antes e após o fogo em ambos os sítios. A amostragem foi realizada em 30 parcelas de 10 $\times 10 \mathrm{~m}$, sendo 15 parcelas em cada sítio. A ocorrência de fogo ocasionou mudanças na composição florística e reduções na riqueza e diversidade de espécies lenhosas mais evidentes no sítio CB (perda de $61 \%$ das espécies) em relação ao SB (perda de 20\% das espécies). Entretanto, o incêndio ocasionou perda relativa de indivíduos $(67,4 \%$ e 73,5\%) e de área basal (75,6\% e 55,2\%) semelhante entre os sítios SB e CB, respectivamente, com exceção dos indivíduos de pequeno porte, os quais foram mais acentuadamente reduzidos no sítio CB. Queimadas em áreas de cerrado com agrupamentos de $A$. verticillatum podem criar condições de retroalimentação positiva e resultar na redução da densidade de indivíduos e na exclusão local de espécies menos tolerantes ao fogo.
\end{abstract}

Palavras-chave: conservação, ecologia do fogo, espécies lenhosas, savana.

\begin{abstract}
The present study aimed to evaluate changes in floristic composition and structure of the woody vegetation after an accidental fire occurred in sites of typical "cerrado" without (SB, control) and with (CB) clumps of Actinocladum verticillatum bamboo in Nova Xavantina, Mato Grosso state. In both sites we sampled all woody individuals with diameter at soil height $\left(\mathrm{Db}_{30}=30 \mathrm{~cm}\right.$ aboveground $) \geq 3 \mathrm{~cm}$, before and after the fire. Sampling was performed in 30 plots of $10 \times 10 \mathrm{~m}, 15$ at each site. The fire caused more evident changes in the floristic composition and reductions in the richness and diversity of woody species in the CB (loss of $61 \%$ of species) than in the SB site (loss of 20\%). However, the fire caused similar relative loss of individuals $(67.4 \%$ and $73.5 \%)$ and basal area (75.6\% and 55.2\%) in SB and CB sites, respectively, with exception of small individuals, which were more markedly reduced in CB site. Fires in cerrado areas occupied with clumps of $A$. verticillatum may create conditions of positive feedback and result in decrease of density and local exclusion of species less tolerant to fire. Key words: conservation, fire ecology, savanna, woody species.
\end{abstract}

\section{Introdução}

A ocorrência de fogo é comum na região do Cerrado, principalmente em função da estacionalidade climática e do acúmulo de combustíveis finos na estação seca (Miranda et al. 2004), o que constitui fator de grande influência sobre a vegetação nativa (Moreira 2000; Hoffmann $\&$ Solbrig 2003). Os efeitos do fogo sobre a vegetação lenhosa em fitofisionomias do Cerrado têm sido bastante estudados, incluindo análises dos danos físicos e de reduções na produtividade de frutos e sementes (Landim \& Hay 1996; Hoffmann 1998; Cirne \& Miranda 2008), do estabelecimento de plântulas (Hoffmann 1996), da reprodução pósfogo (Hoffmann 1998), das taxas de mortalidade (Medeiros \& Miranda 2005; 2008) e dos padrões de dinâmica de populações e comunidades (Hoffmann \& Solbrig 2003; Moreira 2000). É conhecido

\footnotetext{
'Universidade de Brasília - UnB, Programa de Pós-graduação em Ciências Florestais, 70919-970, Brasília, DF, Brasil, Brasil.

${ }^{2}$ Universidade de Brasília -UnB, Programa de Pós-graduação em Ecologia, 70910-900, Brasília, DF, Brasil.

${ }^{3}$ UNEMAT, Depto. Ciências Biológicas, Campus de Nova Xavantina, C.P. 08, 78690-000, Nova Xavantina, MT, Brasil.

${ }^{4}$ Autor para correspondência: biamarimon@hotmail.com
} 
que as espécies lenhosas de formações savânicas de Cerrado apresentam resistência ao fogo por possuírem ritidomas espessos e corticosos, presença de órgãos subterrâneos de reserva e alta capacidade de rebrota (Furley \& Ratter 1988; Hoffmann et al. 2000; Miranda et al. 2002). No entanto, nem todas as espécies apresentam a mesma resistência aos incêndios, o que pode promover mudanças na composição florística e estrutura das comunidades lenhosas, a depender da intensidade e frequência dos incêndios (Moreira 2000; Miranda et al. 2002).

Em formações savânicas e campestres do Cerrado, a maior parte da biomassa que serve de combustível para o fogo na estação seca é composta por gramíneas (Miranda et al. 2004), abundantemente presentes em tais fitofisionomias (Ribeiro \& Walter 2008). Estudos conduzidos por D'Antonio \& Vitousek (1992) e Hoffmann et al. (2004), mostraram que o aumento da densidade de gramíneas e consequentemente de biomassa seca, resulta em incêndios de maior intensidade, causando assim maior mortalidade das espécies lenhosas. No entanto, ainda são desconhecidos os efeitos de queimadas sobre a composição florística, a estrutura e a dinâmica da vegetação de Cerrado em áreas com densos agrupamentos de espécies nativas de bambu.

Os poucos trabalhos conduzidos até o momento indicaram que agrupamentos de bambus em ambientes tropicais podem alterar a germinação de sementes (Silveira 2001), o estabelecimento de plântulas (Silveira 2001; Sanquetta et al. 2006) ou a composição florística e a estrutura da vegetação lenhosa (Silvério et al. 2010). Além disso, os bambus produzem grande biomassa, representando um incremento no material combustível durante a estação seca (Soderstrom 1981). Assim, o efeito integrado do adensamento de bambus e da intensidade do fogo pode representar ameaça adicional à composição florística e estrutura da vegetação em áreas nativas de Cerrado.

No Parque Municipal do Bacaba, município de Nova Xavantina-MT, ocorrem pequenos, isolados e densos agrupamentos da espécie arbustiva de bambu Actinocladum verticillatum (Nees) McClure ex. Soderstr. (Poaceae), em formações savânicas, como o cerrado típico, e florestais, como o cerradão (Silvério et al. 2010) e a floresta de galeria (Marimon et al. 2010). De acordo com Mendonça et al. (2008), A. verticillatum é uma espécie nativa do bioma Cerrado, entretanto na presença de distúrbios, como incêndios recorrentes, os processos demográficos de suas populações podem ser afetados, resultando em incremento de sua área de abrangência e densidade local (Soderstrom 1981; Filgueiras \& Pereira 1988). Desse modo, queimadas em áreas de cerrado com presença de A. verticillatum podem produzir mecanismos de retroalimentação positiva em função do aumento da inflamabilidade e consequentemente da intensidade de novas queimadas.

Neste contexto, o objetivo do presente estudo foi descrever as mudanças na composição florística, na riqueza, na diversidade e na estrutura da vegetação lenhosa após queimada acidental ocorrida em dois sítios de cerrado típico com o mesmo histórico de queimadas nos últimos 20 anos: um ocupado por bambu (Actinocladum verticillatum) e outro sem a presença de bambu, em Nova Xavantina, leste do estado de Mato Grosso. As hipóteses testadas foram: 1) após a queimada, o sítio ocupado por agrupamentos de bambu (CB) apresenta maiores mudanças na composição florística e maior redução da riqueza e diversidade de espécies lenhosas em relação ao sítio não ocupado pelo bambu (SB); 2) após a queimada, o sítio $\mathrm{CB}$ apresenta mudanças estruturais da vegetação lenhosa (morte de indivíduos e perda em área basal) mais evidentes em relação ao sítio sem agrupamentos de bambu (SB).

\section{Material e Métodos}

O estudo foi conduzido em área de cerrado típico (sensu Ribeiro \& Walter 2008) no Parque Municipal do Bacaba (14²' $28,8^{\prime \prime}$ S e 52 $21^{\circ}$ '03,9' W), uma unidade de conservação com área total de 492 hectares e altitude média de $348 \mathrm{~m}$, situada no município de Nova Xavantina-MT. O Parque apresenta várias fitofisionomias e está situado no leste Matogrossense em área de tensão ecológica entre os biomas Cerrado e Floresta Amazônica (Marimon et al. 2006; Silvério et al. 2010). O solo da área estudada, classificado como Latossolo Amarelo, é predominantemente distrófico, ácido, álico, profundo, bem drenado, de textura média e com reduzidos níveis de cálcio e magnésio nas camadas superficiais (Marimon-Junior \& Haridasan 2005). O clima da região é do tipo $A w$ de Köppen, apresentando dois períodos bem definidos, sendo o seco entre maio e setembro e o chuvoso entre outubro e março (Marimon et al. 2001). Segundo dados da Estação Meteorológica localizada no Parque Municipal do Bacaba $\left(9^{\circ}\right.$ Distrito de Meteorologia, Ministério da Agricultura, INMET 
83319-MT), a precipitação média anual calculada em um período de 15 anos foi de aproximadamente $1.536 \mathrm{~mm}$, com médias anuais de temperatura em torno de $25^{\circ} \mathrm{C}$ (Marimon \& Felfili 2006).

Actinocladum verticillatum é uma espécie de bambu nativa do Cerrado (Mendonça et al. 2008) que cresce em touceiras de até quatro metros de altura. A espécie é heterofílica, apresentando folhas grandes, largas e em número reduzido no ápice e nos colmos e folhas verticiladas, estreitas e numerosas situadas em complementos de ramos lateralmente ao nó (Filgueiras 1988). De acordo com Soderstrom (1981), A. verticillatum possui rizomas cobertos por duras escamas que a protegem contra o dessecamento e o fogo, o que maximiza sua capacidade de reprodução vegetativa e garante a produção de novos caules aéreos após a ocorrência de queimadas.

Para a realização deste estudo, foram estabelecidas 30 parcelas permanentes e contíguas de $10 \times 10 \mathrm{~m}$ em área de cerrado típico, sendo 15 em um sítio sem a presença de bambu (SB) e 15 em um sítio com cobertura de bambu superior a 90\% (CB). Os sítios SB e CB distam 20 m entre si (Silvério et al. 2010) e estão sujeitos ao mesmo regime de fogo há 20 anos, o qual tem ocorrido em intervalos periódicos de aproximadamente cinco anos. O uso de parcelas contíguas neste estudo é devido ao fato de o mesmo constituir a continuidade do trabalho realizado por Silvério et al. (2010), o qual teve suas parcelas permanentes estabelecidas em função da ocorrência agrupada do bambu. Desta forma, a ocorrência de incêndio acidental na área gerou a oportunidade de avaliar, de forma inédita, os efeitos do bambu sobre a dinâmica pós-fogo da vegetação lenhosa de cerrado típico, apesar do desenho amostral não contar com réplicas verdadeiras.

No primeiro inventário, realizado em junho de 2008, todos os indivíduos lenhosos com diâmetro de base medido a $30 \mathrm{~cm}$ do solo $\left(\mathrm{Db}_{30}\right) \geq 3 \mathrm{~cm}$ tiveram o diâmetro medido, foram identificados e receberam plaquetas numeradas (Silvério et al. 2010). Em setembro de 2008, cerca de dois meses após a realização do primeiro inventário, ocorreu uma queimada acidental na área de cerrado típico, provavelmente iniciada por proprietários de fazendas vizinhas, o que levou ao desenvolvimento do presente estudo. Em dezembro de 2008, três meses após a queimada, as 30 parcelas foram reavaliadas e todos os indivíduos lenhosos sobreviventes e mortos foram reamostrados. Foram considerados mortos todos os indivíduos com mortalidade completa da parte aérea, sendo observada para esta constatação a ausência de rebrotas, de floema vivo e a perda da casca com exposição do caule.

A amostragem realizada após a queimada seguiu a mesma metodologia empregada na ocasião do primeiro inventário, conduzido antes da queimada (Silvério et al. 2010). A identificação do material botânico foi realizada por meio de comparações com o acervo do Herbário NX (Universidade do Estado de Mato Grosso - UNEMAT) e por consultas a bibliografias e especialistas. O sistema de classificação adotado foi o APG III - Angiosperm Phylogeny Group (APG 2009).

A diversidade de espécies lenhosas de ambos os sítios ( $\mathrm{SB}$ e $\mathrm{CB}$ ) foi calculada para os inventários realizados antes (Silvério et al. 2010) e depois da queimada empregando-se o índice de Shannon (H', na base neperiana). Para comparar os valores do índice de diversidade entre os inventários realizados antes e depois do fogo, dentro de cada sítio, utilizou-se o teste $t$ de Hutcheson (Zar 1999). A similaridade florística foi avaliada entre os sítios (SB e CB) comparando-se os pares de inventários realizados antes e os pares de inventários realizados depois do fogo. Para a análise, foi utilizado o índice qualitativo de Sørensen (CCs) e o índice quantitativo de Morisita (IM) (Wolda 1981).

A riqueza de espécies registrada antes e após a queimada, dentro de cada sítio (SB e CB), foi analisada aplicando-se o método de curvas de rarefação de espécies (Kindt \& Coe 2005). A análise foi realizada com base na aleatorização das amostras empregando-se 1.000 aleatorizações (Kindt \& Coe 2005). Os resultados da acumulação de espécies associados ao desvio padrão de estimativa, para cada sítio antes e depois do fogo, foram apresentados de forma gráfica (Kindt \& Coe 2005). Também por meio do método de rarefação, comparou-se a riqueza de espécies entre os inventários, dentro de cada sítio, empregando-se o programa EcoSim 7.0 (Gotelli \& Entsminger 2001), o qual permite fixar em uma amostra um número de indivíduos menor do que o realmente observado, para efeito de ajuste do esforço amostral. Assim, a riqueza foi estimada para o inventário realizado antes do fogo com a mesma densidade de indivíduos que ficaram vivos no segundo inventário, após o fogo. A partir da média de espécie e variância fornecida pelo programa EcoSim foi calculado um valor de " $p$ " com base no teste $Z$ de distribuição normal padronizada (Zar 1999). 
Para descrever a estrutura da vegetação de ambos os sítios foram contabilizados os indivíduos vivos antes do fogo e os sobreviventes e mortos após o fogo nos dois sítios. A partir de tais informações, foram calculadas as áreas basais de indivíduos vivos antes e de sobreviventes e mortos após o fogo, para cada espécie e para a comunidade de cada sítio (SB e CB). Com base nos dados brutos provenientes dos inventários realizados antes (Silvério et al. 2010) e depois do fogo foram calculadas as perdas absolutas em área basal para as espécies e para a comunidade de cada sítio ( $\mathrm{SB}$ e $\mathrm{CB}$ ), além das porcentagens de indivíduos mortos e de perda em área basal para a comunidade de cada sítio. O cálculo da porcentagem de perda em área basal foi efetuado considerando-se $\mathrm{a}$ área basal dos indivíduos mortos e o decremento (quebra ou perda parcial de tronco) em área basal dos sobreviventes. As porcentagens de indivíduos mortos e de perda em área basal após a ocorrência do fogo foram comparadas entre as comunidades de cada sítio (CB e SB) por meio de testes de Quiquadrado (Zar 1999).

A distribuição dos indivíduos em classes de diâmetro foi efetuada com base na fórmula $\mathrm{A} / \mathrm{K}$ indicada por Spiegel (1976), onde $A$ representa a amplitude dos valores de diâmetro e $K$ representa uma constante definida pelo algoritmo de Sturges, cuja fórmula é $1+3,3 \times \log 10$ n, onde $n$ é o número total de indivíduos amostrados. Para comparar as distribuições dos inventários conduzidos antes e após a ocorrência do fogo, dentro de cada sítio, empregou-se o teste de Kolmogorov-Smirnov (Zar 1999). Com exceção da comparação da riqueza de espécies, todas as análises foram realizadas utilizandose os pacotes vegan e BiodiversityR do programa $\mathrm{R}$ (The R Project for Statistical Computing 2011).

\section{Resultados}

Antes da queimada foram registradas 93 espécies lenhosas no sítio sem bambu (SB) e 64 no sítio com bambu (CB) (Tab. 1; Silvério et al. 2010). Após a queimada, a riqueza dos sítios SB e CB foi de 74 e 25 espécies (Tab. 1), o que representou perdas de 19 (20\% do total) e 39 (61\%) espécies nos sítios SB e CB, respectivamente. Considerando as dez espécies mais abundantes de cada sítio, observou-se que a sobrevivência pós-fogo variou entre 3,3 e $65 \%$ no sítio $\mathrm{SB}$ e 0 e $90 \%$ no sítio CB. No sítio $\mathrm{SB}$, as espécies que apresentaram maior sobrevivência foram Syagrus flexuosa $\left(65 \%\right.$ de sobrevivência e $8^{\mathrm{a}}$ em abundância de indivíduos) e Davilla elliptica $\left(39,2 \%\right.$ e $\left.3^{\mathrm{a}}\right)$ e as que apresentaram menor sobrevivência foram Myrcia lanuginosa $\left(3,3 \% \mathrm{e} 7^{\mathrm{a}}\right)$ e Matayba guianensis $\left(3,8 \%\right.$ e $\left.2^{\mathrm{a}}\right)$ (Tab. 1). No sítio CB Qualea grandiflora ( $8^{\mathrm{a}}$ mais abundante) e Diospyros hispida $\left(10^{\mathrm{a}}\right)$ não apresentaram indivíduo sobrevivente e C. americana ( $90 \%$ e $4^{\mathrm{a}}$ em abundância) e $S$. flexuosa $\left(71,4 \%\right.$ e 1 $\left.{ }^{\mathrm{a}}\right)$ apresentaram maior sobrevivência (Tab. 1).

No sítio SB, dentre as 19 espécies que apresentaram $100 \%$ de mortalidade após a queimada, 11 eram representadas por apenas um indivíduo e oito apresentaram entre dois e seis indivíduos, ao passo que no sítio $\mathrm{CB}$, dentre as 39 espécies que apresentaram $100 \%$ de mortalidade, 13 eram representadas por um indivíduo e 26 entre dois e oito indivíduos (Tab. 1). Adicionalmente, a similaridade florística entre os sítios $\mathrm{SB}$ e CB amostrados antes do fogo foi mais elevada, tanto em termos qualitativos $(\mathrm{CCs}=0,77)$ quanto quantitativos $(\mathrm{IM}=0,63)$ quando comparada à similaridade após a queimada (CCs $=0,46 \mathrm{e} \mathrm{IM}=0,48)$.

No sítio $\mathrm{SB}$, as curvas de riqueza registradas antes e após o fogo indicam que a diferença observada entre inventários está relacionada à diferença na densidade de indivíduos (Fig. 1a). Por outro lado, não há sobreposição das curvas para o sítio $\mathrm{CB}$, o que evidencia menor riqueza de espécies no inventário conduzido após a ocorrência do fogo (Fig. 1b). Considerando o mesmo número de indivíduos, para efeito de ajuste do esforço amostral, no primeiro inventário (antes do fogo) realizado no sítio SB foi estimada riqueza de 73 $\pm 8,8$ espécies em 309 indivíduos, não diferindo da riqueza observada no segundo inventário, após a ocorrência do fogo (74 espécies) $(z=0,457$; $p=0,100)$. Em contrapartida, para o sítio CB foi estimada riqueza de $38,6 \pm 6,4$ espécies em 68 indivíduos antes da queimada, sendo esta riqueza maior que a observada após a ocorrência do fogo ( 25 espécies) $(z=-2,125 ; p=0,016)$.

A diversidade de espécies lenhosas calculada para o sítio SB antes $(3,98)$ (Silvério et al. 2010) e após o fogo $(3,89)$ não diferiu $\left(t_{0,05}=1,19 ; p>0,05\right)$. Em contrapartida, no sítio $\mathrm{CB}$ a diversidade de espécies do inventário realizado antes do fogo $(3,7)$ (Silvério et al. 2010) foi maior que aquela encontrada para o mesmo sítio após o fogo $(2,91)\left(t_{0,05}=9,45 ; p<0,05\right)$.

Antes do fogo foram registrados 947 indivíduos vivos no sítio SB e 257 no sítio CB (Tab. 1; Silvério et al.2010). Após o fogo, constatou-se a morte de 638 indivíduos no SB e de 189 indivíduos no $\mathrm{CB}$, o que resultou em $67,4 \%$ de mortalidade 
Tabela 1 - Alterações nos parâmetros estruturais das espécies lenhosas em sítios sem e com agrupamentos de Actinocladum verticillatum antes e após incêndio em cerrado típico, Nova Xavantina-MT, onde NA/ABA e ND/ABD representam o número de indivíduos e a área basal $\left(\mathrm{m}^{2} / \mathrm{ha}\right)$ antes e depois do fogo, respectivamente. Espécies dispostas em ordem decrescente do NA no sítio sem bambu.

Table 1 - Structural changes of the woody species for sites with and without clumps of Actinocladum verticillatum before and after fire in a typical cerrado, Nova Xavantina-MT, where NA/ABA and ND/ABD represent the number of individuals and basal area $\left(\mathrm{m}^{2} / \mathrm{ha}\right)$ before and after fire, respectively. Species in descending order of NA in the site without bamboo.

\begin{tabular}{|c|c|c|c|c|c|c|c|c|c|}
\hline \multirow{2}{*}{ Espécie } & \multirow{2}{*}{ Família } & \multicolumn{4}{|c|}{ Sítio sem bambu } & \multicolumn{4}{|c|}{ Sítio com bambu } \\
\hline & & NA & ND & $\mathbf{A B A}$ & ABD & NA & ND & ABA & ABD \\
\hline $\begin{array}{l}\text { Diplopterys pubipetala (A.Juss.) W.R.Anderson } \\
\text { \& C.C.Davis }\end{array}$ & Malpighiaceae & 76 & 15 & 7,13 & 0,29 & - & - & - & - \\
\hline Matayba guianensis Aubl. & Sapindaceae & 52 & 2 & 3,24 & 0 & 2 & - & 0 & - \\
\hline Davilla elliptica A.St.-Hil. & Dilleniaceae & 51 & 20 & 7,25 & 1,47 & 1 & - & 0 & - \\
\hline Myrcia splendens (Sw.) DC. & Myrtaceae & 49 & 13 & 6,36 & 2,37 & 4 & - & 0,07 & - \\
\hline Roupala montana Aubl. & Proteaceae & 49 & 12 & 6,35 & 0,82 & 9 & 1 & 0,33 & 0 \\
\hline Antonia ovata Pohl & Loganiaceae & 34 & 10 & 1,66 & 0,18 & 6 & - & 0,06 & - \\
\hline Myrcia lanuginosa $\mathrm{O}$. Berg. & Myrtaceae & 30 & 1 & 1,15 & 0 & - & - & - & - \\
\hline Syagrus flexuosa (Mart.) Becc. & Arecaceae & 29 & 19 & 2,5 & 1,17 & 14 & 10 & 0,60 & 0,28 \\
\hline Erythroxylum suberosum A.St.-Hil. & Erythroxylaceae & 24 & 6 & 1,21 & 0,08 & 3 & - & 0,01 & - \\
\hline Vatairea macrocarpa (Benth.) Ducke & Fabaceae & 24 & 7 & 2,13 & 0,57 & 10 & 2 & 0,35 & 0,01 \\
\hline Mimosa laticifera Rizzini \& A.Mattos & Fabaceae & 21 & 8 & 1,57 & 0,24 & 5 & - & 0,05 & - \\
\hline Byrsonima pachyphylla A.Juss. & Malpighiaceae & 19 & 14 & 0,96 & 0,51 & 4 & 2 & 0,07 & 0,02 \\
\hline Eriotheca gracilipes (K.Schum.) A.Robyns & Malvaceae & 19 & 10 & 1,38 & 0,6 & 5 & 5 & 0,75 & 0,70 \\
\hline Guapira graciliflora (Mart. ex Schmidt) Lundell & Nyctaginaceae & 19 & 8 & 1,21 & 0,51 & 4 & - & 0,03 & - \\
\hline Byrsonima coccolobifolia Kunth & Malpighiaceae & 17 & 10 & 1,17 & 0,54 & 2 & 2 & 0,05 & 0,05 \\
\hline Heisteria ovata Benth. & Olacaceae & 17 & 3 & 0,55 & 0,04 & 3 & - & 0,01 & - \\
\hline Cordiera sessilis (Vell.) Kuntze & Rubiaceae & 16 & 2 & 0,32 & 0 & 2 & - & 0 & - \\
\hline Ouratea spectabilis (Mart.) Engl. & Ochnaceae & 16 & 3 & 0,64 & 0,04 & 7 & - & 0,11 & - \\
\hline Guapira sp. & Nyctaginaceae & 15 & 8 & 0,58 & 0,24 & 4 & 1 & 0,03 & 0,01 \\
\hline Kielmeyera rubriflora Cambess. & Clusiaceae & 15 & 9 & 0,78 & 0,32 & 3 & - & 0,06 & - \\
\hline Terminalia argentea Mart. & Combretaceae & 15 & 3 & 0,77 & 0,08 & 11 & 4 & 0,76 & 0,16 \\
\hline Aspidosperma multiflorum A.DC. & Apocynaceae & 14 & 5 & 0,29 & 0,08 & 1 & - & 0 & - \\
\hline Qualea grandiflora Mart. & Vochysiaceae & 14 & 4 & 0,5 & 0,08 & 8 & - & 0,18 & - \\
\hline Ouratea hexasperma (A.St.-Hil.) Baill. & Ochnaceae & 13 & 3 & 0,33 & 0,05 & 3 & 1 & 0,02 & 0 \\
\hline Anacardium occidentale L. & Anacardiaceae & 11 & 5 & 0,42 & 0,13 & 6 & 2 & 0,44 & 0,05 \\
\hline Rourea induta Planch. & Connaraceae & 11 & 1 & 0,13 & 0 & 2 & - & 0,01 & - \\
\hline Emmotum nitens (Benth.) Miers. & Icacinaceae & 10 & 7 & 1,49 & 1,24 & 13 & 6 & 4,51 & 2,38 \\
\hline Salvertia convallariodora A.St.-Hil. & Vochysiaceae & 10 & 7 & 1,15 & 0,67 & 4 & 4 & 0,43 & 0,41 \\
\hline Buchenavia tomentosa Eichler & Combretaceae & 9 & 6 & 0,72 & 0,44 & 8 & 2 & 0,51 & 0,06 \\
\hline Diospyros hispida A.DC. & Ebenaceae & 9 & 1 & 0,16 & 0 & 7 & - & 0,11 & - \\
\hline Erythroxylum daphnites Mart. & Erythroxylaceae & 9 & 3 & 0,14 & 0,02 & 2 & - & 0,01 & - \\
\hline Guapira noxia (Netto) Lundel & Nyctaginaceae & 9 & 3 & 0,44 & 0,08 & 6 & 2 & 0,43 & 0,05 \\
\hline
\end{tabular}




\begin{tabular}{|c|c|c|c|c|c|c|c|c|c|}
\hline \multirow{2}{*}{ Espécie } & \multirow{2}{*}{ Família } & \multicolumn{4}{|c|}{ Sítio sem bambu } & \multicolumn{4}{|c|}{ Sítio com bambu } \\
\hline & & NA & ND & ABA & ABD & NA & ND & $\mathbf{A B A}$ & ABD \\
\hline Magonia pubescens A.St.-Hil. & Sapindaceae & 9 & 4 & 0,31 & 0,09 & 5 & 2 & 0,18 & 0,09 \\
\hline Qualea parviflora Mart. & Vochysiaceae & 9 & 6 & 0,45 & 0,29 & 3 & - & 0,05 & - \\
\hline Connarus suberosus Planch. & Connaraceae & 8 & 1 & 0,09 & 0 & 2 & - & 0,01 & - \\
\hline Hymenaea stigonocarpa Mart. ex Hayne & Fabaceae & 8 & 2 & 0,15 & 0,03 & 3 & - & 0,02 & - \\
\hline Plathymenia reticulata Benth. & Fabaceae & 8 & 3 & 0,24 & 0,05 & 2 & - & 0,02 & - \\
\hline Protium heptaphyllum (Aubl.) Marchand & Burseraceae & 8 & 3 & 0,14 & 0,03 & 5 & - & 0,03 & - \\
\hline Caryocar brasiliense Cambess. & Caryocaraceae & 7 & 1 & 0,13 & 0 & 6 & 3 & 0,33 & 0,08 \\
\hline Astronium fraxinifolium Schott & Anacardiaceae & 6 & 4 & 0,2 & 0,14 & 1 & 1 & 0,02 & 0,02 \\
\hline Curatella americana $\mathrm{L}$. & Dilleniaceae & 6 & 5 & 0,29 & 0,29 & 10 & 9 & 1,47 & 1,35 \\
\hline Erythroxylum engleri O.E.Schulz & Erythroxylaceae & 6 & 1 & 0,1 & 0,01 & - & - & - & - \\
\hline Pouteria ramiflora (Mart.) Radlk. & Sapotaceae & 6 & 3 & 0,09 & 0,03 & 1 & - & 0,01 & - \\
\hline Tachigali vulgaris L.G.Silva \& H.C.Lima & Fabaceae & 6 & 0 & 0,09 & 0 & - & - & - & - \\
\hline Dipteryx alata Vogel & Fabaceae & 5 & 2 & 0,07 & 0,02 & 1 & - & 0 & - \\
\hline Leptolobium dasycarpum Vogel & Fabaceae & 5 & 1 & 0,07 & 0,01 & - & - & - & - \\
\hline Mezilaurus crassiramea (Meisn.) Taub. ex Mez & Lauraceae & 5 & 3 & 0,15 & 0,05 & 2 & - & 0,01 & - \\
\hline Mouriri elliptica Mart. & Melastomataceae & 5 & 3 & 0,09 & 0,04 & 1 & - & 0 & - \\
\hline Myrcia camapuanensis N.Silveira & Myrtaceae & 5 & 0 & 0,05 & 0 & - & - & - & - \\
\hline Neea theifera Oerst. & Nyctaginaceae & 5 & 1 & 0,07 & 0,01 & 2 & - & 0,01 & - \\
\hline Qualea multiflora Mart. & Vochysiaceae & 5 & 1 & 0,06 & 0 & 8 & 2 & 0,13 & 0,01 \\
\hline Alibertia edulis (Rich.) A.Rich. & Rubiaceae & 4 & 1 & 0,03 & 0 & - & - & - & - \\
\hline Annona coriacea Mart. & Annonaceae & 4 & 1 & 0,03 & 0,01 & 6 & - & 0,11 & - \\
\hline Bowdichia virgilioides Kunth & Fabaceae & 4 & 4 & 0,07 & 0,06 & 4 & 1 & 0,09 & 0,02 \\
\hline Heteropterys byrsonimiifolia A.Juss. & Malpighiaceae & 4 & 2 & 0,03 & 0,01 & 4 & - & 0,04 & - \\
\hline Kielmeyera coriacea Mart. \& Zucc. & Clusiaceae & 4 & 1 & 0,03 & 0 & 2 & 2 & 0,02 & 0,02 \\
\hline Lafoensia pacari A.St.-Hil & Lythraceae & 4 & 0 & 0,03 & 0 & 4 & 1 & 0,04 & 0,01 \\
\hline Luehea candicans Mart. \& Zucc. & Malvaceae & 4 & 1 & 0,07 & 0,02 & 1 & - & 0 & - \\
\hline Pseudobombax longiflorum (Mart. \& Zucc.) A.Robyns & Malvaceae & 4 & 1 & 0,05 & 0,01 & 1 & - & 0 & - \\
\hline Aspidosperma tomentosum Mart. & Apocynaceae & 3 & 1 & 0,04 & 0,01 & 5 & 1 & 0,10 & 0 \\
\hline Dimorphandra mollis Benth. & Fabaceae & 3 & 1 & 0,05 & 0,01 & 5 & - & 0,08 & - \\
\hline Euplassa inaequalis (Pohl) Engl. & Proteaceae & 3 & 2 & 0,06 & 0,03 & - & - & - & - \\
\hline Guettarda virburnoides Cham. \& Schltdl. & Rubiaceae & 3 & 0 & 0,01 & 0 & - & - & - & - \\
\hline Handroanthus ochraceus (Cham.) Mattos & Bignoniaceae & 3 & 2 & 0,12 & 0,05 & - & - & - & - \\
\hline Himatanthus obovatus (Müll.Arg.) Woodson & Apocynaceae & 3 & 0 & 0,01 & 0 & - & - & - & - \\
\hline Luetzelburgia praecox (Harms) Harms & Fabaceae & 3 & 2 & 0,02 & 0,01 & 2 & 1 & 0,02 & 0 \\
\hline Machaerium acutifolium Vogel & Fabaceae & 3 & 1 & 0,04 & 0,01 & 1 & - & 0,01 & - \\
\hline Myrcia tomentosa (Aubl.) DC. & Myrtaceae & 3 & 1 & 0,01 & 0 & 1 & - & 0 & - \\
\hline Psidium sp. & Myrtaceae & 3 & 0 & 0,02 & 0 & 1 & - & 0,01 & - \\
\hline Styrax ferrugineus Ness \& Mart. & Styracaceae & 3 & 2 & 0,03 & 0,01 & - & - & - & - \\
\hline
\end{tabular}




\begin{tabular}{|c|c|c|c|c|c|c|c|c|c|}
\hline \multirow{2}{*}{ Espécie } & \multirow{2}{*}{ Família } & \multicolumn{4}{|c|}{ Sítio sem bambu } & \multicolumn{4}{|c|}{ Sítio com bambu } \\
\hline & & NA & ND & $\mathbf{A B A}$ & ABD & NA & ND & $\mathbf{A B A}$ & ABD \\
\hline Tocoyena formosa (Cham. \& Schltdl.) K.Schum. & Rubiaceae & 3 & 1 & 0,01 & 0 & - & - & - & - \\
\hline Andira cujabensis Benth. & Fabaceae & 2 & 1 & 0,03 & 0,01 & - & - & - & - \\
\hline Aspidosperma macrocarpon Mart. & Apocynaceae & 2 & 1 & 0,01 & 0 & - & - & - & - \\
\hline Aspidosperma subincanum Mart. & Apocynaceae & 2 & 2 & 0,01 & 0,01 & - & - & - & - \\
\hline Casearia sylvestris $\mathrm{Sw}$. & Salicaceae & 2 & 0 & 0,01 & 0 & - & - & - & - \\
\hline Hirtella glandulosa Spreng. & Chrysobalanaceae & 2 & 2 & 0,03 & 0,03 & - & - & - & - \\
\hline Licania humilis Cham. \& Schltdl. & Chrysobalanaceae & 2 & 0 & 0,01 & 0 & - & - & - & - \\
\hline Peltogyne confertiflora (Mart. ex Hayne) Benth. & Fabaceae & 2 & 1 & 0,02 & 0,01 & - & - & - & - \\
\hline Simarouba versicolor A.St.-Hil. & Simaroubaceae & 2 & 2 & 0,02 & 0,02 & - & - & - & - \\
\hline Tabebuia aurea (Silva Manso) Benth. \& Hook.f. ex S.Moore & Bignoniaceae & 2 & 2 & 0,01 & 0,01 & 1 & - & 0 & - \\
\hline Bauhinia brevipes Vogel & Fabaceae & 1 & 0 & 0 & 0 & - & - & - & - \\
\hline Brosimum gaudichaudii Trécul & Moraceae & 1 & 0 & 0 & 0 & - & - & - & - \\
\hline Cochlospermum regium (Mart. ex Schrank) Pilg. & Cochlospermaceae & 1 & 0 & 0 & 0 & - & - & - & - \\
\hline Cybianthus detergens Mart. & Myrsinaceae & 1 & 0 & 0 & 0 & - & - & - & - \\
\hline Dalbergia miscolobium Benth. & Fabaceae & 1 & 0 & 0 & 0 & 2 & - & 0,01 & - \\
\hline Eremanthus sp. & Asteraceae & 1 & 0 & 0,01 & 0 & - & - & - & - \\
\hline Eugenia aurata O.Berg & Myrtaceae & 1 & 0 & 0 & 0 & - & - & - & - \\
\hline Fridericia cinnamomea (DC.) L.C.Lohmann & Bignoniaceae & 1 & 0 & 0 & 0 & - & - & - & - \\
\hline Himatanthus sucuuba (Spruce ex Müll.Arg.) Woodson & Apocynaceae & 1 & 1 & 0,01 & 0,01 & - & - & - & - \\
\hline Piptocarpha sp. & Asteraceae & 1 & 0 & 0 & 0 & - & - & - & - \\
\hline Schefflera distractiflora (Harms) Frodin & Araliaceae & 1 & 0 & 0 & 0 & - & - & - & - \\
\hline Vochysia rufa Mart. & Vochysiaceae & 1 & 1 & 0,01 & 0,01 & 1 & - & 0 & - \\
\hline Xylopia aromatica (Lam.) Mart. & Annonaceae & 1 & 0 & 0,01 & 0 & - & - & - & - \\
\hline Couepia grandiflora (Mart. \& Zucc.) Benth. & Chrysobalanaceae & - & - & - & - & 1 & - & 0 & - \\
\hline Myrcia sp. & Myrtaceae & - & - & - & - & 2 & - & 0,01 & - \\
\hline Pterodon pubescens (Benth.) Benth. & Fabaceae & - & - & - & - & 2 & 1 & 0,02 & 0,01 \\
\hline TOTAL & & 947 & 309 & 58,4 & 14,3 & 257 & 68 & 12,9 & 5,78 \\
\hline
\end{tabular}

no SB e 73,5\% no CB (Tab. 1), valores que não diferiram $\left(\chi_{1 ; 0,05}^{2}=1,924 ; p=0,165\right)$.

Aárea basal registrada antes do fogo no sítio SB $\left(58,4 \mathrm{~m}^{2}\right)$ e no sítio CB (12,9 m²) (Silvério et al. 2010) apresentou perdas respectivas de $75,6 \%$ e $55,2 \%$ depois do fogo, resultando em áreas basais de 14,27 $\mathrm{m}^{2}$ e $5,78 \mathrm{~m}^{2}$ nos sítios $\mathrm{SB}$ e $\mathrm{CB}$, respectivamente (Tab. 1). Os percentuais de perda em área basal, entretanto, não diferiram entre os sítios $\mathrm{SB}$ e $\mathrm{CB}$ $\left(\chi_{1 ; 0,05}^{2}=3,20 ; p=0,076\right)$ após a ocorrência do fogo.

Antes do fogo, a primeira classe de diâmetros (3 a $5,5 \mathrm{~cm}$ ) era representada por
583 indivíduos vivos no sítio SB (61,5\% do total) e 108 indivíduos no CB (42\%) (Silvério et al. 2010). No entanto, após a queimada houve redução evidente no número de indivíduos nessa classe diamétrica em ambos os sítios (487 indivíduos ou 83,53\% no sítio SB e 99 indivíduos ou $91,67 \%$ no CB) (Fig. 2a-b). Em função desta redução, a distribuição dos indivíduos em classes de diâmetro diferiu entre os inventários realizados antes e depois do fogo, tanto para o sítio SB (KS, $\left.\chi^{2}=88,22 ; \mathrm{p}<0,01\right)$ quanto para o $\mathrm{CB}\left(\mathrm{KS}, \chi^{2}=\right.$ $18,08 ; \mathrm{p}<0,01)$. 

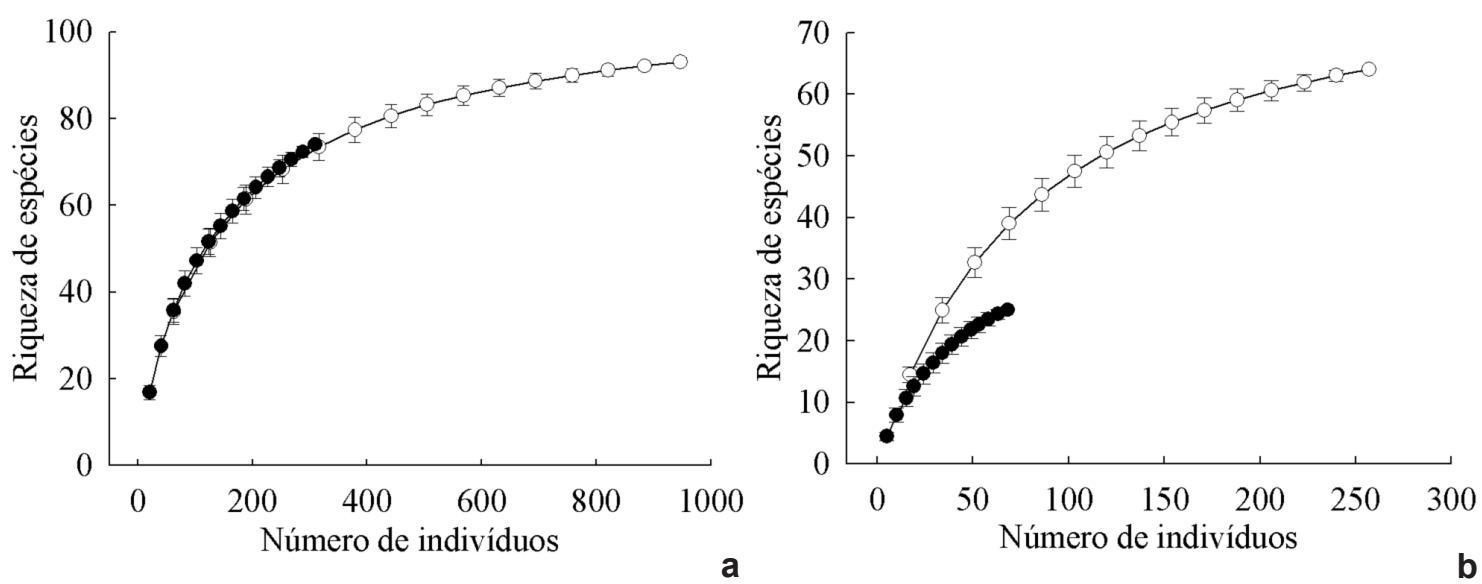

Figura 1 - Curvas de rarefação de espécies baseadas na aleatorização das amostras para os sítios sem (a) e com (b) agrupamentos de Actinocladum verticillatum antes (-০-) e após (-๑-) incêndio em cerrado típico, Nova XavantinaMT. Pontos centrais representam médias e barras verticais representam desvios padrão.

Figure 1 - Species rarefaction curves based on randomization of samples for sites with (a) and without (b) clumps of Actinocladum verticillatum before (-O-) and after (-๑-) fire in a typical cerrado, Nova Xavantina-MT. Central points represent means and vertical bars represent standard deviation.

\section{Discussão}

A ocorrência do fogo ocasionou maiores mudanças florísticas e reduções na riqueza e diversidade de espécies no sítio $\mathrm{CB}$ em relação ao $\mathrm{SB}$, indicando maior severidade dos efeitos do fogo para a vegetação lenhosa em ambientes com a presença dos agrupamentos de Actinocladum verticillatum. Reforçando esta conclusão, a análise de rarefação evidenciou que no sítio $\mathrm{CB}$ a diferença na densidade de indivíduos não foi suficiente para explicar a perda de espécies, havendo assim efetiva redução da riqueza neste sítio após o fogo, independentemente da relação entre esforço amostral e número de espécies registradas (Colwell \& Coddington 1994). Miranda et al. (1993) observaram que a intensidade do fogo está diretamente relacionada com a quantidade de combustível fino acumulado. No caso do cerrado típico estudado, a ocupação do bambu A. verticillatum certamente representa grande incremento da biomassa inflamável na época seca, como já destacado por Soderstrom (1981) e sugerido por Silvério et al. (2010).

Desse modo, queimadas em áreas de cerrado com agrupamentos de Actinocladum verticillatum, cuja capacidade de rebrota e crescimento após eventos de fogo é elevada (Soderstrom 1981), podem criar condições de retroalimentação positiva, ou seja, podem favorecer queimadas mais frequentes e com maior severidade. Este processo, por sua vez, pode resultar em expressiva redução da densidade de indivíduos e consequentemente na exclusão local de espécies menos tolerantes ao fogo, como é o caso de Qualea grandiflora e Diospyros hispida, que estavam entre as 10 espécies mais abundantes e tiveram todos os indivíduos mortos após o fogo (Tab. 1). Por outro lado, este processo pode favorecer competitivamente espécies tolerantes ou indiferentes ao fogo, como ocorrido para Curatella americana (Tab. 1), modificando assim a composição e a estrutura da vegetação, como observado por Moreira (2000) estudando diferentes fitofisionomias de Cerrado no Distrito Federal.

Além disso, a diferença na abundância de indivíduos de várias espécies entre os sítios $\mathrm{SB}$ e $\mathrm{CB}$ antes do fogo pode ter contribuído para a maior redução da riqueza no sítio $\mathrm{CB}$ após o fogo. Silvério et al. (2010) mostraram que entre as 64 espécies comuns a ambos os sítios antes do fogo, $42(65 \%)$ apresentavam densidade de indivíduos mais elevada no sítio $\mathrm{SB}$ em relação ao $\mathrm{CB}$, tal como constatado para Diplopterys pubipetala, Matayba guianensis, Davilla elliptica, Myrcia splendens, Roupala montana, entre outras. Neste caso, o efeito mais severo do fogo associado à baixa densidade das espécies pode contribuir para que muitas espécies desapareçam do referido sítio, pois a morte de alguns indivíduos pode representar a exclusão local da espécie. Assim, áreas de cerrado 


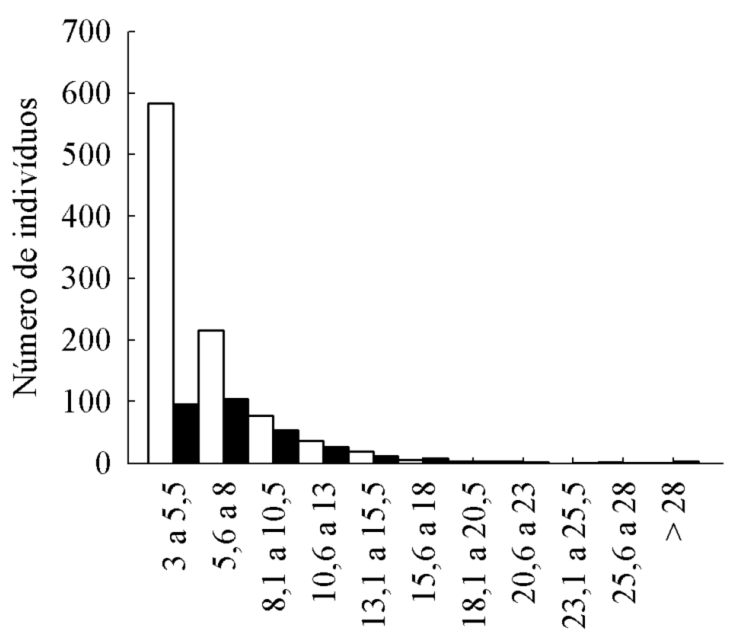

Classes de diâmetro $(\mathrm{cm})$

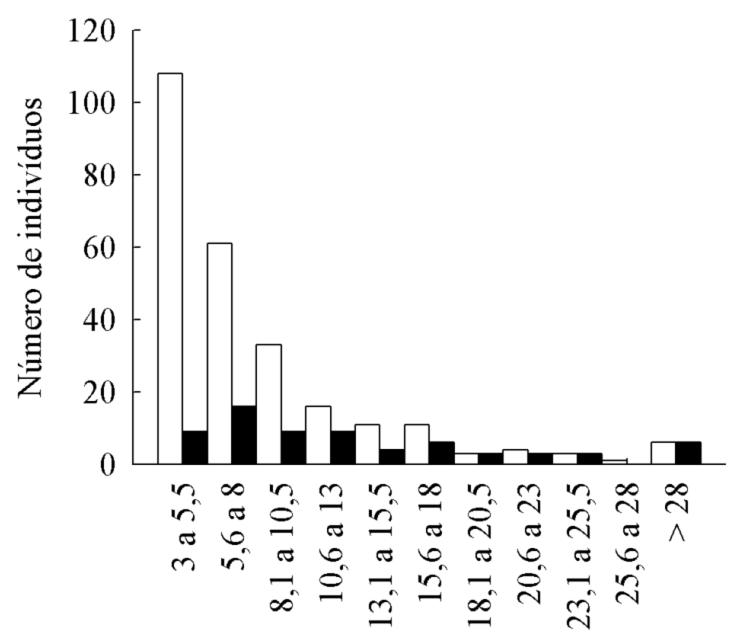

Classes de diâmetro $(\mathrm{cm})$

a

Figura 2 - Número de indivíduos por classe de diâmetro para os sítios sem (a) e com (b) agrupamentos de Actinocladum verticillatum antes $(\square)$ e após ( $\square$ ) incêndio em cerrado típico, Nova Xavantina-MT.

Figure 2 - Number of individuals per diameter classes for sites with (a) and without (b) clumps of Actinocladum verticillatum before $(\square)$ and after ( $\square$ ) fire in a typical cerrado, Nova Xavantina-MT.

ocupadas por esta espécie de bambu podem apresentar perdas consideráveis de biodiversidade (Silvério et al. 2010), especialmente se o regime natural das queimadas for alterado.

Os processos já discutidos indicam que a presença do bambu associada à passagem do fogo pode resultar na formação de fisionomias com menores riquezas e densidades de espécies lenhosas. Ademais, as maiores mudanças florísticas observadas no sítio CB promoveram o desenvolvimento de duas comunidades com composição florística diferenciada, o que foi evidenciado pela redução da similaridade florística entre os sítios SB e CB após a queimada. Moreira (2000) já havia observado evidentes efeitos do fogo na composição de espécies e na estrutura da vegetação, entretanto, o presente estudo indicou que as mudanças pós-fogo podem ser ainda mais drásticas em áreas com elevada cobertura de Actinocladum verticillatum.

Em termos estruturais relativos, as perdas de indivíduos e de área basal após o incêndio foram semelhantes para ambos os sítios. Neste caso, este resultado pode estar relacionado à maior suscetibilidade de indivíduos menores aos efeitos do fogo (Moreira 2000; Miranda et al. 2002), já que antes do fogo o sítio SB apresentava $61,5 \%$ dos indivíduos na primeira classe de diâmetros (3 a $5,5 \mathrm{~cm}$ ), enquanto no sítio $\mathrm{CB}$ esta proporção era 20\% menor (Fig. 2a-b). Assim, a elevada mortalidade de indivíduos de pequeno porte no sítio SB (Fig. 2a) pode ter contribuído para que a mortalidade fosse semelhante em ambos os sítios, apesar da aparente maior severidade do fogo no sítio CB. Por outro lado, o sítio $\mathrm{CB}$, que já apresentava menor proporção de indivíduos de pequeno porte (Silvério et al. 2010), provavelmente apresentou menor perda relativa em função da maior representatividade de indivíduos maiores, que são mais resistentes ao fogo (Frost \& Robertson 1987; Brando et al. 2011).

Considerando a amostragem realizada antes do fogo, a distribuição dos indivíduos nas classes de diâmetro foi semelhante entre os sítios $\mathrm{SB}$ e CB (Fig. 2a-b), apresentando a maioria dos indivíduos na primeira classe (Fig. 2a-b, Silvério et al. 2010). No entanto, após a queimada tal distribuição foi mais afetada no sítio $\mathrm{CB}$, uma vez que neste sítio houve redução mais acentuada na proporção de indivíduos na primeira classe de diâmetros em comparação com o sítio SB (Fig. 2a-b). Apesar de Silvério et al. (2010) terem sugerido que o estabelecimento de novos indivíduos lenhosos no sítio $\mathrm{CB}$ poderia estar sendo comprometido por diferentes mecanismos relacionados à competição interespecífica com o bambu, os resultados do presente estudo sugerem que 
o fogo também pode atuar como mecanismo promotor de divergências estruturais entre áreas ocupadas e não ocupadas pelo bambu.

Segundo Frost \& Robertson (1987) queimadas em savanas têm chamas cujas alturas médias variam entre 0,8 e $2,8 \mathrm{~m}$. No sítio SB do cerrado aqui estudado, as chamas atingiram alturas entre 1 e $2 \mathrm{~m}$ (obs. pessoal), enquanto no sítio CB, em função da altura das touceiras do bambu que atingem em média $4 \mathrm{~m}$ (obs. pessoal; Filgueiras 1988), as chamas alcançaram entre 3 e $4 \mathrm{~m}$ (obs. pessoal). Neste caso, chamas mais altas podem ter causado maiores danos à parte aérea viva das espécies lenhosas, resultando em maior densidade de mortos no sítio CB.

Ambas as hipóteses formuladas não devem ser rejeitadas, pois o conjunto dos resultados do presente estudo aponta maiores mudanças estruturais e na composição florística, além de reduções na riqueza e diversidade de espécies lenhosas após o fogo no sítio $\mathrm{CB}$ quando comparado ao sítio SB. Ressalta-se a necessidade da condução de estudos avaliando, em longo prazo, os efeitos conjugados das queimadas e dos aumentos nas densidades populacionais de Actinocladum verticillatum sobre a resiliência das comunidades lenhosas de cerrado, incluindo principalmente descrições sobre a sobrevivência e o estabelecimento de novos indivíduos. Tais estudos podem nortear estratégias de monitoramento da expansão populacional do bambu $A$. verticillatum e de manejo do fogo para a manutenção da composição, estrutura e diversidade florística de áreas nativas.

\section{Agradecimentos}

À CAPES, a concessão de bolsas de estudo aos dois primeiros autores e o apoio financeiro através do projeto PROCAD. Aos Biólogos Edmar A. Oliveira, Leandro M. dos Santos e Claudinei O. Santos, o importante auxílio durante as etapas de campo. Ao CNPq, o apoio financeiro (Projeto PELD - Transição Cerrado-Floresta Amazônica: bases ecológicas e sócio-ambientais para a conservação - Proc. No 558069/2009-6).

\section{Referências}

Angiosperm Phylogeny Group - APG. 2009. An update of the Angiosperm Phylogeny Group classification for the orders and families of flowering plants: APG III. Botanical Journal of the Linnean Society 161: 105-121.
Brando, P.M.; Nepstad, D.C.; Balch, J.K.; Bolker, B.; Christman, M.C.; Coe, M.T. \& Putz, J.E. 2011. Fire-induced tree mortality in a neotropical forest: The roles of bark traits, tree size, wood density, and fire behavior. Global Change Biology 18: 630-641.

Cirne, P. \& Miranda, H.S. 2008. Effects of prescribed fires on the survival and release of seeds of Kielmeyera coriacea (Spr.) Mart. (Clusiaceae) in savannas of central Brazil. Brazilian Journal of Plant Physiology 20: 197-204.

Colwell, R.K. \& Coddington, J.A. 1994. Estimating terrestrial biodiversity through extrapolation. Philosophical Transactions of the Royal Society of London, B 345: 101-118.

D’Antonio, C.M. \& Vitousek, P.M. 1992. Biological invasions by exotic grasses, the grass/fire cycle, and global change. Annual Review of Ecology and Systematics 23: 63-87.

Filgueiras, T.S. 1988. Native forage grass species from the Federal District, Brazil (Gramineae: Bambusoideae). Revista Brasileira de Botânica 11: 47-66.

Filgueiras, T.S. \& Pereira, B.A.S. 1988. On the flowering of Actinocladum verticillatum (Gramineae: Bambusoideae). Biotropica 20: 164-166.

Frost, P.H.G., Robertson, F. 1987. The ecological effects of fire in savannas. In: Walker, B.H. (ed.). Determinants of tropical savannas. IRL Press Limited, Oxford. Pp. 93-139

Furley P.A. \& Ratter J.A. 1988. Soil resource and plant communities of the central Brazilian cerrado and their development. Journal of Biogeography 15: 97-108.

Gotelli, N.J. \& Entsminger, G.L. 2001. EcoSim: Null models software for ecology. Version 7.0. Acquired Intelligence Inc. \& Kesey-Bear.

Hoffmann, W.A. 1996. The effects of fire and cover on seedlings establishment in a neotropical savanna. Journal of Ecology 84: 383-393.

Hoffmann, W.A. 1998. Post-burn reproduction of woody plants in a Neotropical Savanna: the relative importance of sexual and vegetative reproduction. Journal of Applied Ecology 35: 422-433.

Hoffmann, W.A.; Bazzaz, F.A.; Chatterton, N.J.; Harrison, P. \& Jackson, R.B. 2000. Elevated $\mathrm{CO}_{2}$ enhances resprouting of a tropical savanna tree. Oecologia 123: 312-317.

Hoffmann, W.A. \& Solbrig, O.T. 2003. The role of topkill in the differential response of savanna woody species to fire. Forest Ecology and Management 180: 273-286.

Hoffmann, W.A.; Lucatelli, V.M.P.C.; Silva, F.J.; Azevedo, I.N.C.; Marinho, M.S.; Albuquerque, A.M.S.; Lopes, A.O. \& Moreira, S.P. 2004. Impact of the invasive alien grass Melinis minutiflora at the savanna-forest ecotone in the Brazilian Cerrado. Diversity and Distributions 10: 99-103.

Kindt, R. \& Coe, R. 2005. Tree diversity analysis: a manual and software for common statistical 
methods for ecological and biodiversity studies. World Agroforestry Centre (ICRAF), Nairobi. 196p.

Landim, M.F. \& Hay, J.D.V. 1996. Impacto do fogo sobre alguns aspectos da biologia reprodutiva de Kielmeyera coriacea Mart. Revista Brasileira de Biologia 56: 127-134.

Marimon, B.S.; Felfili, J.M. \& Haridasan, M. 2001. Studies in monodominant forests in eastern Mato Grosso, Brazil: I. A forest of Brosimum rubescens Taub. Edinburgh Journal of Botany 58: 123-137.

Marimon, B.S. \& Felfili, J.M. 2006. Chuva de sementes em uma floresta monodominante de Brosimum rubescens Taub. e em uma floresta mista adjacente no Vale do Araguaia, MT, Brasil. Acta Botanica Brasilica 20: 423-432.

Marimon, B.S.; Lima, E.S.; Duarte, T.G.; Chieregatto, L.C. \& Ratter, J.A. 2006. Observations on the vegetation of northeastern Mato Grosso, Brazil. IV. An analysis of the Cerrado-Amazonian Forest ecotone. Edinburgh Journal of Botany 63: 323-341.

Marimon, B.S.; Felfili, J.M.; Lima, E.S.; Duarte, W.M.G. \& Marimon-Junior, B.H. 2010. Environmental determinants for natural regenerations of gallery forest at the Cerrado/Amazonia boundaries in Brazil. Acta Amazonica 40: 107-118.

Marimon-Junior, B.H. \& Haridasan, M. 2005. Comparação da vegetação arbórea e características edáficas de um cerradão e um cerrado stricto sensu em áreas adjacentes sobre solo distrófico no leste de Mato Grosso, Brasil. Acta Botanica Brasilica 19: 913-926.

Medeiros, M.B. \& Miranda, H.S. 2005. Mortalidade pósfogo em espécies lenhosas de campo sujo submetido a três queimadas prescritas anuais. Acta Botanica Brasilica 19: 493-500.

Medeiros, M.B. \& Miranda, H.S. 2008. Post-fire resprouting and mortality in cerrado woody plant species over a three-year period. Edinburgh Journal of Botany 65: 1-16.

Mendonça, R.C.; Felfili, J.M.; Walter, B.M.T.; Silva Júnior, M.C.; Rezende, A.V.; Filgueiras, T.S.; Nogueira, P.E. \& Fagg, C.W. 2008. Flora vascular do bioma Cerrado. In: Sano, S.M.; Almeida, S.P.; Ribeiro, J.F. (eds.). Cerrado: ecologia e flora. Embrapa Informação Tecnológica, Brasília. Pp. 421-1.279

Miranda, A.C.; Miranda, H.S.; Dias, I.F.O. \& Dias, B.F.S. 1993. Soil and air temperatures during prescribed cerrados fires in Central Brazil. Journal of Tropical Ecology 9: 313-320.
Miranda, H.S.; Bustamante, M.M.C. \& Miranda, A.C. 2002. The fire factor. In: Oliveira, P.S., Marquis, R.J. (eds.). The Cerrados of Brazil: ecology and natural history a Neotropical Savanna. Columbia University Press, New York. Pp. 51-68.

Miranda, H.S.; Sato, M.N.; Andrade, S.M.A.; Haridasan, M. \& Morais, H.C. 2004. Queimadas de Cerrado: caracterização e impactos. In: Aguiar, L.M.S., Camargo, A.J.A. (eds.). Cerrado: ecologia e caracterização. Embrapa Cerrados, Brasília. Pp. 69-123.

Moreira, A.G. 2000. Effects of fire protection on savanna structure in Central Brazil. Journal of Biogeography 27:1021-1029.

R Development Core Team. 2011. R: A language and environment for statistical computing. R Foundation for Statistical Computing, Vienna. Disponível em $<$ http://www.R-project.org/>Acesso em 1 Out 2011.

Ribeiro, J.F. \& Walter, B.M.T. 2008. As principais fitofisionomias do Bioma Cerrado. In: Sano, S.M.; Almeida, S.P. \& Ribeiro, J.F. (eds.). Cerrado: ecologia e flora. Embrapa Informação Tecnológica, Brasília. Pp.151-212.

Sanquetta, C.R.; Dalla Corte, A.P.; Vulcanis, L.; Berni, D.M. \& Biscaia, A.G. 2006. Estabelecimento de plântulas de espécies arbóreas em um experimento de controle de taquaras (Bambusoideae) no sul do Paraná, Brasil. Revista Ciências Exatas e Naturais 8: 163-177.

Silveira, M. 2001. A floresta aberta com bambu no sudoeste da Amazônia: padrões e processos em múltiplas escalas. Tese de Doutorado. Universidade de Brasília, Brasília. 121p.

Silvério, D.V.; Mews, H.A.; Lenza, E. \& Marimon, B.S. 2010. Impactos do agrupamento do bambu Actinocladum verticillatum (Ness) McClure ex. Sodertr. (Poaceae) sobre a vegetação lenhosa de duas fitofisionomias de Cerrado na transição CerradoFloresta Amazônica. Acta Amazonica 40: 347-355.

Soderstrom, T.R. 1981. Observations on fire-adapted bamboo of the Brazilian Cerrado, Actinocladum verticillatum (Poaceae: Bambusoidae). American Journal of Botany 68: 1200-1211.

Spiegel, M.P. 1976. Estatística. McGraw-Hill, São Paulo.

Wolda, H. 1981. Similarity indices, sample size and diversity. Oecologia 50: 296-302.

Zar, J.H. 1999. Biostatistical analysis. Prentice Hall, New Jersey. $663 p$. 